\title{
Electrochemical Methods for Manufacturing Silver Nanoparticles
}

\author{
Sitti Rahmah \\ Faculty of Fisheries and Marine Science \\ Universitas Borneo \\ Tarakan, Indonesia \\ sittirahmah25@gmail.com,
}

\author{
Fredy Kurniawan \\ Faculty of Mathematics and Natural Sciences \\ Institut Teknologi Sepuluh November \\ Surabaya, Indonesia
}

\begin{abstract}
In this study the manufacture of silver nanoparticles has been carried out using an electrochemical method. An electrochemical method by electrolysis is one of the alternatives of making silver nanoparticles. It is easy, simple and does not require a long time. Silver nanoparticle characterization using Ultraviolet Visible (UV-Vis) and X-ray Diffraction (XRD). The test results showed that the concentrations of $0.2 \mathrm{M}$ sodium citrate have a wavelength of 416-418nm and the absorbance of 0.825 . XRD tests showed the peaks characteristic in $2 \Theta$ of $38.06^{\circ}, 44.14^{\circ}, 64.47^{\circ}$, and $77.40^{\circ}$.
\end{abstract}

Keywords-Electrochemical, Silver Nanoparticle, UltravioletVisible; $x$-ray diffraction

\section{INTRODUCTION}

Nanoparticles are clusters of atoms in the size range of 1-100 nm. "Nano" is a Greek word synonymous to dwarf meaning extremely small. Nanoparticles are widely used in the present century as they have the defined chemical, optical and mechanical properties [1].

Synthesis of silver nanoparticles can be done by several methods such as electrochemical methods [2] photochemistry [3], laser ablation [4], thermal decomposition [5]. Factors that affect the synthesis of silver nanoparticles are solution temperature, concentration of precursors, reducing agents and reaction time [6].

Preparation of the highlights of colloidal silver nanoparticles is by use of a reducing agent. There are a wide variety of reducing agents that can be used, one of which, is sodium citrate. Sodium citrate excellence in the synthesis of silver nanoparticles is that it can be functioned as reducing, stabilizing and complexing agents [7]. Besides it is relatively cheap.

An electrochemical method by electrolysis is one of the alternatives in the manufacturing of silver nanoparticles because of the lower processing temperatures, the simple equipment used, and the controllable products and the short time needed. Electrochemical methods have previously used a metal silver to produce silver nanoparticles by using a reducing agent Polyvinylpyrrolidone (PVP) [8]

The main advantage of an electrochemical method lies on the high purity and particle size of the nanoparticles that can be controlled by adjusting the current density without being supported by the expensive equipment or vacuum. Key to the success of an electrochemical method is in the right choices of chemical substances and of processing conditions [9].
In this study, the synthesis of silver nanoparticles is conducted through an electrochemical method by the electrolysis to take advantage of sodium citrate as the reducing agents to produce silver nanoparticles. This needs a relatively affordable cost. The resulted silver nanoparticles are then expected to be applicable in the various fields such as cosmetics [10], the catalyst [11], and glucose sensor [12].

\section{METHOD}

Equipments needed are power supply, multimeter, cable, alligator clamps, heater, magnetic stirrer, hot cables of $1 \mathrm{~mm}$, Silicon Carbide Sandpaper, slass, pipette volume, drop pipette, Volumetric flask, spatula of metal silver material, sodium citrate, distilled water. Instruments are the Ultraviolet Visible (UV-Vis) and X-ray Diffraction (XRD).

In preparation, Silver Nanoparticles Silver nanoparticles are synthesized using an electrolysis cell with a set of silver electrodes as the anode and cathode. The electrolyte material used is water while the reducing agent used is $0.2 \mathrm{M}$ sodium citrate. The potential value used is 10 volts and a synthesis time is 30 minutes. The electrolysis device of the first step is set and the power supply is turned on. Furthermore, $400 \mathrm{ml}$ of distilled water is boiled and maintained at a temperature of $60^{\circ} \mathrm{C}$. Then, $10 \mathrm{~mL}$ of 0.2 $\mathrm{M}$ sodium citrate is added to the distilled water and stirred using a magnetic stirrer.

The observed color changes from colorless to yellow. The yellow color shows silver nanoparticles have been formed. The uptake of silver nanoparticles can be determined through characterizing it using ultraviolet rays. The uptake of silver colored nanoparticle is about 400500nm [6] [13].

\section{RESULTS AND DISCUSSION}

Spectrophotometer ultraviolet visible (UV-VIS) absorption has been proved to be quite sensitive to the formation of silver colloids because silver nanoparticles exhibit an intense absorption peak due to the surface Plasmon (it describes the collective excitation of conduction electrons in a metal) excitation [14]. UV-visible spectroscopy is one of the most widely used techniques for structural characterization of silver nanoparticles. Spectra UV Vis can be seen in figure 1 . 


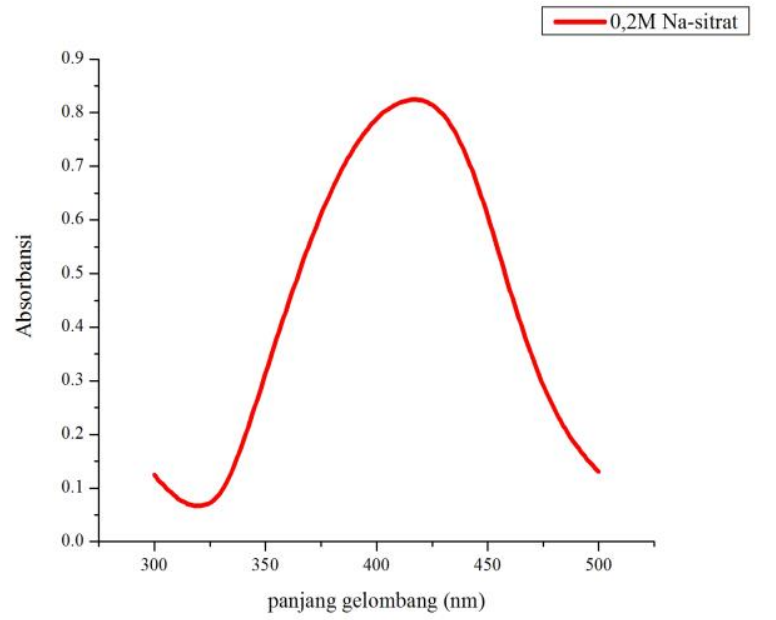

Fig. 1 Ultraviolet visible spectra

Fig.1 shows the UV-VIS spectra of the silver colloid in the range of $300 \mathrm{~nm}-500 \mathrm{~nm}$. Plasmon peak at $445 \mathrm{~nm}$ ) is typical for silver nanoparticles. Absorbance value provide information on the number of particles while the wavelength provides information size particle [6].

The higher the absorbance value assumes the more nanoparticles formed. Stability of colloid of silver nanoparticles can be seen from the changes in the peak absorbance. Stability of colloidal solution of silver nanoparticles can be seen from the changes in the peak absorption. If a shift to the peak absorption is higher, this indicates that the silver nanoparticle colloidal solution is less stable due to occurrence of agglomeration. In the case of agglomeration, the color of the solution changed and wavelength of absorption will shift [6].

Silver nanoparticle formation was observed in 5 days. Color formed is yellow. Yellow is the color characteristics of the silver nanoparticles [15] [13] [2].

$\mathrm{X}$-ray diffraction in this study is only used to identify the crystal phase. XRD analysis of test results can be seen in Fig.2.

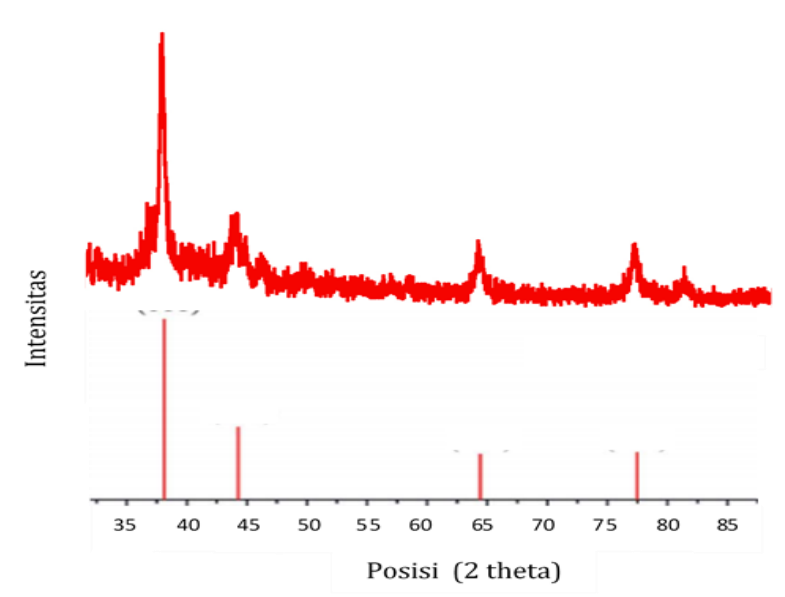

Fig. 2 X-ray diffraction spectra

Fig. 2 shows the XRD spectra. XRD Spectra determines a product phase. Part (a) shows the standard of XRD Ag (JCPDS no. 04-0783). While the image of silver nanoparticles $b$ test results. Diffraction peaks at position 2 $\Theta$ of $38.060,44.140,64.470$, and 77.400. Diffraction of peak product is not much different from the standard diffraction and that of the previous studies [2] [16]. So it can be confirmed that the product is Ag. The XRD pattern also shows that the product has good crystallinity and high purity.

\section{IV.CONCLUSION}

Based on this research, it can be concluded that the electrochemical method can be used for manufacturing silver nanoparticles. Excellent electrochemical electrolysis method that is easy, simple and does not require a long time.

\section{ACKNOWLEDGMENT}

Thanks to Dr.rer.nat Fredy Kurniawan, friends of Analytical Science Laboratory research Institute of Technology Sepuluh Nopember Surabaya and to the Faculty of Fisheries and Marine Science University of Borneo Tarakan for prayer, support and cooperation.

\section{REFERENCES}

[1] G. P, L. H, W. K, H. J and T. W, "Preparation and antibacterial activity of Fe3O4@Ag nanoparticles.," Nanotechnology., vol. 18, pp. 604-611, 2007.

[2] Z. Huang, H. Jiang, P. Liu, J. Sun, D. Guo, J. Shan, and N. Gu. , "Continuous synthesis of size-tunable silver nanoparticles by a green electrolysis method and multi-electrode design for high yield.," J Mater Chem A, vol. 3, no. 5, pp. 1925-1929, 2015.

[3] Z. Li, Y. Li, X.-F. Qian, J. Yin, and Z.-K. Zhu. , " A simple method for selective immobilization of silver nanoparticle.," Appl. Surf. Sci., vol. 250, no. 1, pp. 109-116, 2005.

[4] T. Tsuji, N. Watanabe, and M. Tsuji. , " Laser induced morphology change of silver colloids: formation of nano-size wires.," Laser induced Appl. Surf. Sci., vol. 211, no. 1, pp. 189-193, 2003.

[5] Y. H. Kim, D. K. Lee, and Y. S. Kang., "Synthesis and characterization of $\mathrm{Ag}$ and $\mathrm{Ag}-\mathrm{SiO} 2$ nanoparticles," Colloids Surf. Physicochem. Eng. Asp., vol. 257, pp. 273-276, 2005.

[6] A. Šileikaitè, I. Prosyčevas, J. Puišo, A. Juraitis, and A. Guobienė , " Analysis of silver nanoparticles produced by chemical reduction of silver salt solution.," Mater Sci-Medzg, vol. 12, pp. 287-291, 2006.

[7] Jiang, X.C., Chen, C.Y., Chen, W.M., Yu, A.B., "Role of Citric Acid in the Formation of Silver Nanoplates through a Synergistic Reduction Approach"," Langmuir, vol. 26, pp. 4400-4408, 2010.

[8] Rashid A Khaydarov, Renat R Khaydarov, Olga Gapurova, Yuri Estrin, and Thomas Sceper, "Electrochemical Methods for Synthesis of Silver Nanoparticles.," J Nanopart Res, vol. 11, pp. 1193-1200, 2009.

[9] Rodriguez-Sanches L, Blanco MC, Lopez-Quitela MA , " Electrochemical synthesis of Silver Nanoparticles.," J Phys Chem $B$, vol. 104, pp. 9683-9688, 2000.

[10] Kokura, S., Handa, O., Takagi, T., Ishikawa, T., Naito, Y., and Yoshikawa, T., "Silver nanoparticles as a safe preservative for use in cosmetics.," Nanomedicine, vol. 6, pp. 570-574, 2010.

[11] N. Pradhan, A. Pal, and T. Pal. , " Silver nanoparticle catalyzed reduction of aromatic nitro compounds.," Colloid Sur. Physicochem. Eng. Asp., vol. 196, no. 2, pp. 247-257, 2002.

[12] Lin, J., He, C., Zhao, Y., and Zhang, S., "One-Step Synthesis of Silver Nanoparticles/Carbon Nanotubes/Chitosan Film and its Application in Glucose Biosensor.," Sensor and Actuators B: Chemical, Vol. 137, hal. 768-773., vol. 137, pp. 768-773, 2009.

[13] Gusman, M. G., Dille, J., and Godet, S., "Synthesis of Silver Nanoparticles by Chemical Reduction Method and their 
Antibacterial Activity," International Journal of Chemical and Biomolecular Engineering, vol. 2, pp. 104-111, 2009.

[14] Gao, X., Gu, G., Hu, Z., Guo, Y., Fu, X., and Song, J., "A Simple Method for Preparation of Silver Dendrites Colloids and Surfaces A," Physicochemical Engineering Aspects, Vols. 57-61, p. 254, 2005.

[15] Solomon, S.D., Bahadory, M., Jeyarajasingam, A.V., Rutkowsky, S.A., and Boritz, C, "Synthesis and Study of Silver Nanoparticles," Journal of Chemical Education, vol. 84, pp. 322-325, 2007.

[16] T. Maneerung, S. Tokura, and R. Rujiravanit, "Impregnation of silver nanoparticles into bacterial cellulose for antimicrobial wound dressing Carbohydr," Polym., vol. 72, no. 1, pp. 43-51, 2008. 